\title{
Early-onset neonatal sepsis caused by Neisseria meningitidis serogroup B: case report and literature review of a 102-year period
}

\author{
Tayyib Mubashar ${ }^{1}$, Paul Christopher Turner ${ }^{2}$, Siba Prosad Paul ${ }^{3 \bullet}$ \\ ${ }^{1}$ Medical School, University of Exeter, Exeter; Departments of ${ }^{2}$ Medical Microbiology and ${ }^{3}$ Paediatric, Torbay Hospital, Torquay, \\ United Kingdom.
}

\begin{abstract}
A 36-week-2-day-old male infant was admitted to the neonatal unit with respiratory distress, hypoglycaemia and suspected early onset neonatal sepsis for respiratory support, monitoring and intravenous antibiotics. His initial C-reactive protein was $12 \mathrm{mg} / \mathrm{L}$, this increased to $66 \mathrm{mg} / \mathrm{L}$ at 24 hours. Blood cultures at $48 \mathrm{hours}$ confirmed Neisseria meningitidis serogroup B. As the isolate was sensitive to benzylpenicillin the same antibiotic was continued for a total of 7 days. His mother remained asymptomatic but was monitored closely. Ciprofloxacin chemoprophylaxis was given to close family contacts. Neisseria meningitidis causing early onset neonatal sepsis is extremely rare and neonates may have minimal symptoms at presentation. A table reviewing all documented cases of early onset neonatal sepsis caused by Neisseria meningitidis over a 102-year time period is included. There is need for early identification and initiation of empirical antibiotic therapy pending confirmation and sensitivities.
\end{abstract}

Key words: neonatal sepsis, Neisseria meningitidis, invasive meningococcal disease, antibiotics, chemoprophylaxis.

Neonatal sepsis is defined as 'a systemic inflammatory response syndrome specifically presenting with abnormalities of temperature and/or leukocytosis in an infant from birth to 4 weeks of age as a result of proven infection'. ${ }^{1}$ It can present as neonatal meningitis, septicaemia or a combination of both. Early onset neonatal sepsis (EOS) presents in the first 6 days of life with late onset neonatal sepsis presenting at 7-28 days. $^{1}$ A prospective multicenter surveillance study involving 12 English neonatal units over 3 years (2006-2008) with 124 confirmed isolates reported the most common organisms responsible for EOS were: Group B Streptococcus (GBS) (50\%), Escherichia coli (18\%), Listeria monocytogenes (6\%), Streptococcus spp. (6\%) and Staphylococcus aureus (5\%). ${ }^{2}$ The same study also reported the incidence of EOS at $0.9 / 1,000$ live births. ${ }^{2}$

$\triangle$ Siba Prosad Paul

siba@doctors.org.uk

Received 11st December 2018; revised 29th January 2019; accepted 24th February 2019
Neisseria meningitidis causes invasive meningococcal disease (IMD). Although rare in developed countries, it remains one of the most feared infectious diseases. A data linkage project by Public Health England estimated the total burden of IMD in England to be 5,115 laboratory-confirmed cases over a five-year period (2007-2011) with group B meningococci responsible for $87.33 \%$ (n: 4,034) of hospitalised cases. ${ }^{3}$ The same study reported that infants $(<1$ year-old) accounted for 1,115/4,619 (24.14\%) of cases, although no specific further breakdown for neonates were given. Neisseria meningitidis as a causative organism in EOS is extremely rare. ${ }^{4,5}$ This article describes a case of early onset neonatal sepsis caused by Neisseria meningitidis.

\section{Case Report}

A 36-week-2-day-old male was born following a normal vaginal delivery, with poor respiratory effort, tachycardia, pallor and hypotonia. The neonate was resuscitated via face mask 
with a T-piece used to deliver intermittent positive pressure ventilation. As the neonate's spontaneous respiratory effort remained inadequate, he was started on positive end expiratory pressure of 5-8 cm water pressure and transferred to the neonatal unit. Initial neonatal blood gases demonstrated a respiratory acidosis ( $\mathrm{pH} 7.11 ; \mathrm{PaCO}_{2} 12 \mathrm{kPa}$ ).

On admission his temperature was $37.3^{\circ} \mathrm{C}$, pulse rate $140 / \mathrm{min}$, respiratory rate $44 / \mathrm{min}$, and blood pressure 59/39 $\mathrm{mmHg}$. Blood glucose on admission was $1.1 \mathrm{mmol} / \mathrm{L}(19.8 \mathrm{mg} / \mathrm{dl})$ and a $10 \%$ dextrose bolus of $2 \mathrm{ml} / \mathrm{kg}$ was administered. No maternal risk factors were identified. However, clinical indicators for possible EOS included: a) altered behaviour/ responsiveness b) tachycardia c) signs of respiratory distress syndrome (RDS) needing CPAP support $d$ ) prematurity e) hypoglycaemia.

Routine blood investigations and blood cultures were obtained, and the neonate was commenced on intravenous benzylpenicillin $(50 \mathrm{mg} / \mathrm{kg} / \mathrm{dose}$ twice daily) and gentamicin $(5 \mathrm{mg} / \mathrm{kg} /$ dose 36 hourly). Chest X-ray demonstrated moderate RDS. C-reactive protein (CRP) was $12 \mathrm{mg} / \mathrm{L}$ increasing to $66 \mathrm{mg} / \mathrm{L}$ at 24 hours; a white blood cell count of $4.6 \times 10^{9} / \mathrm{L}$ increasing to $13.2 \times 10^{9} / \mathrm{L}$. Blood gases improved as did blood glucose. He was weaned off CPAP at 24 hours.

CSF samples obtained at 31 hours of age showed no evidence of meningitis. Blood cultures at 48 hours identified Neisseria meningitidis serogroup B. Following a discussion with a medical microbiologist and as the neonate was improving clinically, penicillin was continued for a total of 7 days, and gentamicin was discontinued after the third dose. Antibiotic sensitivities confirmed that the organism was susceptible to penicillin [minimum inhibitory concentration (MIC) $0.06 \mathrm{mg} / \mathrm{L}]$ and cefotaxime (MIC $0.004 \mathrm{mg} / \mathrm{L}$ ). His CRP had settled to 9 $\mathrm{mg} / \mathrm{L}$ on day-5.

Close contact chemoprophylaxis with ciprofloxacin was prescribed for the parents and grandparents. The neonate was discharged at day-12 and reported as doing well at clinic follow-up few months later. Participation involved informed consent.

\section{Discussion}

The isolate from the neonate was Neisseria meningitidis group B type 1 (subtype NT/NT). IMD in neonates has been caused by all major serogroups of Neisseria meningitidis such as A, B, C, $\mathrm{Y}$ and W135. ${ }^{4-8}$ However, literature reviews highlight the preponderance of Neisseria meningitidis serogroup B as the major causative agent in neonatal IMD. ${ }^{4,6-9}$

Koplick, in 1916, published the first case of neonatal meningococcal meningitis (NMM). ${ }^{9}$ The exact incidence of Neisseria meningitidis as a causative agent for neonatal sepsis is not well known. ${ }^{6}$ A review article reported 15/424 (3.5\%) cases of confirmed neonatal bacterial meningitis in England and Wales that were due to Neisseria meningitidis over a 5-year period (1985-87, 199697). ${ }^{10} \mathrm{~A}$ more recent study from France between 2001 and 2013 found that 23/831 (2.8\%) cases of neonatal bacterial meningitis were caused by Neisseria meningitidis. ${ }^{9}$

A literature review conducted using the PubMed, Google Scholar, and Scirus databases revealed 21 published cases of EOS caused by Neisseria meningitidis over a 102-year period (1916 - 2018). ${ }^{4,6,8,9,11}$ Table I highlights the clinicopathological outcomes of 16 cases of EOS where adequate records were available. The 16 cases of EOS caused by Neisseria meningitidis were due to NMM (n: 7), septicaemia (n: 5) or a combination of both (n: 3). The mortality rate in neonates from EOS caused by Neisseria meningitidis was $40 \%(6 / 15)$ based on this literature review over the 102-year period.

A review of the literature by Kiray Baş et al. ${ }^{4}$ identified significant risk factors for neonatal meningococcaemia which included: prematurity, maternal acute viral infections, functional asplenia, crowded environments, maternal smoking or neonates exposed to passive tobacco smoking. EOS cases caused 


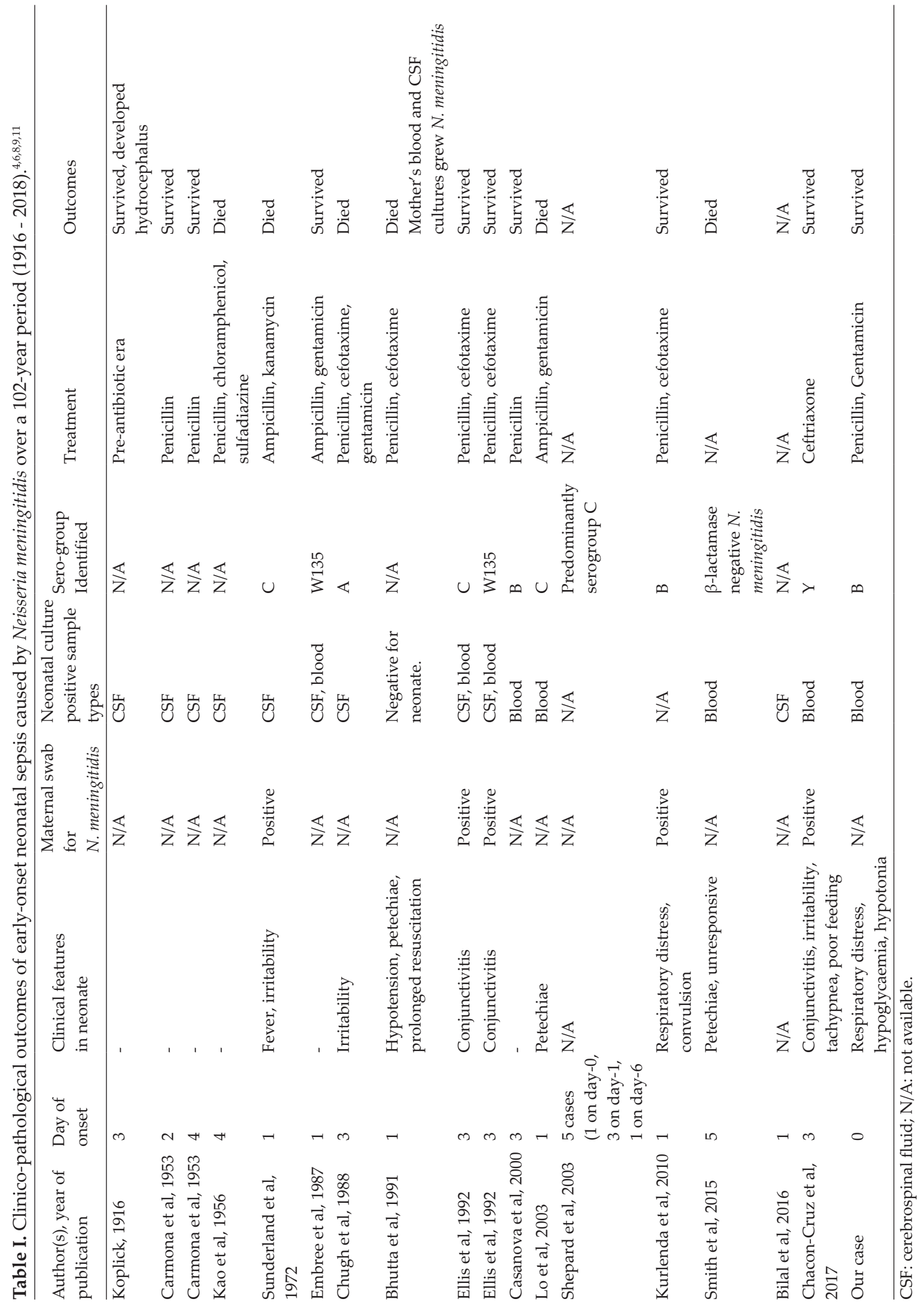


by Neisseria meningitidis may have minimal symptoms as was the cases in $6 / 15$ babies or may have minimal symptoms at presentation. ${ }^{4}$ It is vital that based on risk factors, red flags and clinical suspicion for IMD, the neonate undergoes early investigations and empirical antibiotic therapy with intravenous benzylpenicillin and gentamicin is initiated. Depending on the response and clinical progress, the antibiotic regime may be changed to cefotaxime or another appropriate antibiotic based on the sensitivity profile. However, cases of meningococcal infection truly resistant to penicillin are extremely rare although the MIC as well as higher dosing need to be kept in mind.

In cases of IMD resulting in EOS, it is important to investigate genitourinary colonization of the mother and consider nasopharyngeal carriage among close contacts. Although the nasopharyngeal carriage rate is high in the community, IMD in neonates remains a rarity, possibly due to the protective effect of maternal antibodies passed on from mother to fetus in-utero. ${ }^{4}$ However, prematurity and low birth weight could have an impact on this. ${ }^{4}$ The availability of the immunizations in the UK against Meningococcal $B$ and $C$ as well as the quadrivalent vaccine could impact on the epidemiology of IMD in the future.

It is important to liaise with Public Heath England or similar national organizations and offer chemoprophylaxis to all close contacts including healthcare professionals who may have come into contact with the neonate's respiratory secretions. ${ }^{4}$ The mother should also be monitored closely and if she becomes unwell or there is any suspicion of maternal sepsis, then blood cultures and appropriate antibiotic therapy e.g. intravenous ceftriaxone should be started promptly.

EOS due to IMD is extremely rare but can be life-threatening. Whilst rare, with symptom manifestation being atypical compared to older age groups, a raised index of suspicion should lead to prompt administration of appropriate intravenous antibiotics as well as taking blood/
CSF cultures as this is likely to be associated with a better outcome. Further research is needed to facilitate a consensus, as currently there are no guidelines for empirical treatment of neonatal IMD.

\section{REFERENCES}

1. NICE (2012). Antibiotics for early-onset neonatal infection: antibiotics for the prevention and treatment of early-onset neonatal infection. Available at: https://www.nice.org.uk/guidance/cg149/evidence/ cg149-antibiotics-for-earlyonset-neonatal-infectionfull-guideline2 (Accessed on 11st December, 2018)

2. Vergnano S, Menson E, Kennea N, et al. Neonatal infections in England: the NeonIN surveillance network. Arch Dis Child Fetal Neonatal Ed 2011; 96: F9-F14.

3. Edge C, Waight P, Ribeiro S, Borrow R, Ramsay M, Ladhani S. Clinical diagnoses and outcomes of 4619 hospitalised cases of laboratory-confirmed invasive meningococcal disease in England: linkage analysis of multiple national databases. J Infect 2016; 73: 427436.

4. Kiray Baş E, Bülbül A, Cömert S, Uslu S, Arslan S, Nuhoglu A. Neonatal infection with Neisseria meningitidis: analysis of a 97-year period plus case study. J Clin Microbiol 2014; 52: 3478-3482.

5. Zhang J, Wang Y, Luo J, Ding Y, Shao F. Meningitis caused by Neisseria meningitides serogroup B in a newborn in China. SOJ Microbiol Infect Dis 2016; 4: 1-3.

6. Shepard CW, Rosenstein NE, Fischer M; Active Bacterial Core Surveillance Team. Neonatal meningococcal disease in the United States, 1990 to 1999. Pediatr Infect Dis J 2003; 22: 418-422.

7. Bosman M, Archary M, Mahabeer P, Bobat R. Early onset meningococcal meningitis. South Afr J Epidemiol Infect 2013; 28: 177-179.

8. Chacon-Cruz E, Alvelais-Palacios JA, RodriguezValencia JA, Lopatynsky-Reyes EZ, VolkerSoberanes ML, Rivas-Landeros RM. Meningococcal neonatal purulent conjunctivitis/sepsis and asymptomatic carriage of $\mathrm{N}$. meningitidis in mother's vagina and both parents' nasopharynx. Case Rep Infect Dis 2017; 2017: 6132857.

9. Bilal A, Taha MK, Caeymaex L, Cohen R, Levy C, Durrmeyer X; Groupe des Pédiatres et microbiologistes de l'Observatoire National des Méningites; Members of the National Reference Center for Meningococci. Neonatal meningococcal meningitis in France from 2001 to 2013. Pediatr Infect Dis J 2016; 35: 1270-1272. 
10. Heath PT, Nik Yusoff NK, Baker CJ. Neonatal meningitis. Arch Dis Child Fetal Neonatal Ed 2003; 88: F173-F178.
11. Smith SM, Roy Babbitt MF, Schwartz A, Gomez MR, Fox MD. Early onset neonatal Neisseria meningitidis meningitis: a case report. EC Paediatr 2015; 1: 35-38. 